\title{
COVID-19: Risks Facing Doctors who Treat Infected Patients
}

Kenneth YY Kok

Pengiran Anak Puteri Rashidah Sa'adatul Bolkiah Institute of Health Sciences, Universiti Brunei Darussalam, Brunei

Keywords

COVID-19, Pandemic, Doctors, Risks

\section{Introduction}

On $31^{\text {st }}$ December 2019, the World Health Organization (WHO) was informed of a cluster of pneumonia cases of unknown aetiology in the city of Wuhan in Hubei Province, China [1]. On $9^{\text {th }}$ January 2020, WHO released a statement on this cluster of cases, which stated that a preliminary determination of a novel (or new) coronavirus were identified in hospitalized persons with pneumonia in Wuhan [2]. The virus was then referred to as 2019-nCoV [3].

Coronaviruses are enveloped RNA viruses found in animals and humans. Currently, 6 coronavirus species are known which cause illnesses in humans. Four of the human coronaviruses, HCoV-229E, HCoV-HKU1, HCoV-NL63 and $\mathrm{HCOV}-\mathrm{OC} 43$ are prevalent and are associated with a range of respiratory outcomes, including bronchitis and pneumonia [4]. The other two, severe acute respiratory syndrome coronavirus (SARS-CoV) and Middle East respiratory syndrome coronavirus (MERS-CoV) are zoonotic in origin and can cause fatalities [5].

Reported cases of 2019-nCoV indicated are that the overall case-fatality is around $2 \%$. An analysis of the first 425 cases provided an estimated mean incubation period of 5.2 days $(95 \% \mathrm{Cl} 4.1-7.0)$ and a basic reproductive number (Ro) of 2.2 (95\% Cl 1.4-3.9) [5]. It has also been reported that people with 2019-nCoV may be infectious before showing any symptoms [6]. However, currently available data indicated that only those who have symptoms are causing the majority of virus spread.

On $30^{\text {th }}$ January 2020, the WHO Director General declared the outbreak of novel coronavirus 2019-nCoV as a Public Health Emergency of International Concern (PHEIC) [7]. WHO advised that all countries be prepared for containment, including active surveillance, early detection, isolation and case management, contact tracing and prevention of onward spread of 2019-nCoV infection, and to share full data with WHO. On $11^{\text {th }}$ February 2020, the WHO renamed 2019-nCoV infection as COVID-19 and formally recognized the virus as a sister to the severe acute respiratory syndrome coronaviruses (SARS-CoVs) of the species Severe acute respiratory syndrome-related coronavirus and designated it as severe acute respiratory syndrome coronavirus 2 (SARS-CoV-2) [8].

Since its emergence in Wuhan, China in late December 2019, the numbers of cases and deaths due to COVID-19 around the world continue to rise. On $11^{\text {th }}$ March 2020, WHO has declared the COVID-19 outbreak a pandemic [9], as the number of cases worldwide now stands at 932,605 with 46,809 deaths (last updated: 2 April 2020).

The complete clinical picture of COVID-19 is still not fully known. Reported illnesses have ranged from very mild such as fever, cough and shortness of breath (including some with no reported symptoms) to severe such as pneumonia, multi-organ failure and death [10]. A series from the Chinese Center for Disease Control and Preventionon 72,314 cases reported that most cases of COVID-19 were classified as mild (81\%). However, $14 \%$ were classified as severe and $5 \%$ were critical [11]. Older individuals and individuals with severe co-morbidities seem to be at risk of developing severe illness [12].

Due to the large number of cases and the health care demand, health care workers (HCWs) are recognized as high-risk group to acquire this infection [13]. In an early series of 138 patients reported from a Wuhan hospital, $29 \%$ of the cases involved were HCWs. Among the affected HCWs, 77.5\% worked on general wards, $17.5 \%$ in the emergency department and $5 \%$ in the intensive care unit [14]. Chinese health authorities and a team from the WHO reported on 24 February 2020 that 3,387 HCWs in China had been infected with COVID-19, more than $90 \%$ of whom were in Hubei province, the outbreak's epicenter [15]. It was also reported that $14.8 \%$ of confirmed cases among HCWs were classified as severe or critical [12]. In other countries with high numbers of COVID-19 infections, HCWs are amongst those who are infected while treating

*Corresponding author: Kenneth YY Kok, Pengiran Anak Puteri Rashidah Sa'adatul Bolkiah Institute of Health Sciences, Universiti Brunei Darussalam, Jalan Tungku Link, BE1410, Brunei

Accepted: April 16, 2020

Published online: April 18, 2020

Citation: Kenneth YYK (2020) COVID-19: Risks Facing Doctors who Treat Infected Patients. Res Rev Infect Dis 3(1):49-52 
patients. In China, at least 26 Chinese HCWs who dedicated themselves to fighting the epidemic have died, including 13 of them who died after contracting COVID-19, according to Chinese news outlet Caixing [16]. Amongst those HCWs who succumbed to COVID-19 were 8 doctors after being infected at work while treating patients. In Italy, 61 doctors and other HCW have died of COVID-19, with 11,591 deaths according to the country's National Federation of Orders of Surgeons and Dentists which was updated on 31 March 2020 [17].

As the pandemic continued, more and more reports are emerging from other affected countries that doctors are dying after being infected with COVID-19 while treating infected patients. In Indonesia, the Indonesian Doctors Association confirmed on $23^{\text {rd }}$ March 2020 that 6 doctors had died from COVID-19 [18], while Spain, France, United Kingdom and the Philippines have also reported the death of doctors from COVID-19 who contracted the infection while treating patients.

\section{Discussion}

Doctors are particularly vulnerable to COVID-19 for a number of reasons.

\section{Highly contagious agent and a greater exposure to the agent}

COVID-19 is highly contagious and medical staffs are exposed to a greater viral load than the general public because of the nature of their work; they interact with many infected patients and perform high risk activities during the course of their daily work.

\section{Unusual presentations}

In the series of 138 patients with COVID 19 reported from Wuhan, China, the most common symptoms at the onset of illness were fever (98.6\%), fatigue (69.6\%), dry cough (59.4\%), myalgia (34.8\%) and dyspnea (31.2\%). Less common symptoms reported were headache, dizziness, abdominal pain, diarrhea, nausea and vomiting. A total of 14 patients $(10.1 \%)$ initially presented with diarrhea and nausea one to two days prior to development of fever and dyspnea [14]. During the initial phase, when the coronavirus first appeared in Wuhan, China, one infected patient was initially sent to the Surgical ward because he displayed atypical symptoms, this patient went on to infect at least 10 HCWs. The unusual presentations could lead to a delay in diagnosis, resulting in the spread of the virus before definitive signs of COVID-19 infection manifest.

\section{Danger of the infection not initially realized}

Doctors are at risk if patients with a highly contagious infection are not identified, this is most likely to happen during the early stages of an outbreak. The doctors may start to treat these infected patients without taking the necessary precautions if patients with COVID-19 are not identified quickly and correctly, which leaves the doctors more exposed to the infection. This is a risk not only to healthcare workers, but also to visitors and other patients at the hospital.

\section{Shortages of essential supplies}

There were chronic shortages of supplies such as masks, suits and protective gears resulting in even more acute shortages of these essential personal protection equipment (PPE) during the COVID-19 outbreak. According to a survey conducted by the union National Nurses United, less than a third of nurses in the US work at health care facilities felt that their facilities had enough PPE to handle an outbreak of COVID-19 [19].

Shortage of essential supplies such as face masks is further compounded by panic buying of these masks by the general public during the outbreak. Face masks do not offer the general public much protection against the spread of COVID-19. Most of time, the masks are not fitted properly and the general public do not know how to put them on and take them off safely. But that has not stopped the public from buying them. According to data from the Nielsen Retail Measurement Services, sales of medical masks in US went up by $319 \%$ in the 4 week period ended on February 22, 2020 [20]. Most countries lack sufficient PPE to respond to a large scale event and this acute shortage of essential supplies puts HCWs further at risk.

\section{Understaffing}

Chronic understaffing of the public hospitals made the medical staff susceptible to the virus when they had to treat an overwhelming number of infected patients during the COVID-19 outbreak. This shortage is further compounded when doctors get sick or have to be quarantined due to exposure to COVID-19.

\section{Lack of experience}

The doctors who treated COVID-19 patients may lack experience of such a contagious infection. When the outbreak initially occurred, doctors from different departments and specialties were mobilized to join the outbreak response team. A lot of these doctors were asked to work outside their area of expertise. Many of them lacked training in PPE, infection control in contaminated environments, close contact with sick patients, and in procedures such as intubation in a high-risk infectious disease environment. In a crisis situation without proper training, doctors rushing to the frontline can easily become infected, become ill and spread infections to others.

\section{Doctors are disproportionately affected}

In past epidemics, doctors and other HCWs were also disproportionately affected by the illnesses they were treating. According to $\mathrm{WHO}$, during the Ebola outbreak in west Africa between 2013 and 2016, where more than 11,000 people died, HCWs were between 21 and 32 times more likely to be infected with Ebola than people in the general adult population [21]. It was also reported that the risk of acquiring Ebola was 100 times higher for HCWs in Sierra Leone than the general population [22]. Wong reported that around $20 \%$ of people who were infected with SARS during that outbreak in 2002 and 2003 were medical workers [23]. 


\section{Overworked}

Exhaustion is another reason why doctors have high vulnerability to infection. On $25^{\text {th }}$ February 2020, The Los Angeles Times reported that $18 \mathrm{HCWs}$ who were involved in the COVID-19 response in China had died not because of infection but because of cardiac arrest or other ailments due to overwork and fatigue; one victim was hit by a car while taking temperatures on a highway. They included doctors who died of overwork and cardiac arrest after treating patients. A clinic deputy head died after working 33 days straight treating COVID-19 patients, a doctor aged 32 died of a heart attack in his dorm room after treating patients and another doctor aged 37 died in his clinic due to fatigue [24]. Doctors in South Korea have also been reported to collapse from exhaustion while treating COVID-19 patients [25].

\section{Extreme mental stress}

Several specific sources of stress for doctors and other HCWs treating patients with COVID-19 during the pandemic have been identified. These include stress related to the need to observe strict biosecurity measures such as the physical strain of wearing PPE, physical isolation and constant vigilance regarding infection control procedures; stress related to medical and personal demands such as workload demands, separation from and concern about family members, fears about infection and its implications for self, patients and family; and stress related to stigma such as others' fear of contact with those treating COVID-19 [26]. It was reported that during the SARS epidemic in 2002, doctors reported facing stigma because they worked with infected patients. The fear, uncertainty and stigma were associated with psychological distress among the doctors [27].

It was also reported that some nurses treating COVID-19 patients in a hospital in Korea resigned, citing overwork and mental stress as reasons for their resignations [25]. Another stressful and anxiety-provoking situation for doctors is when the doctors have to care for their colleagues and to see them become patients and getting ill from the infection. All these mental stresses add to the physical stress and exhaustion treating patient infected with COVID-19.

In view of the challenges faced by doctors treating patients with COVID-19, various international agencies, government departments and medical professional organizations have issued guidelines to hospitals and individual HCWs on hospital preparedness and the management of COVID-19 patients. These guidelines include training of health care professionalsand identification of patients; measures on infections prevention and control, patient movement, hand hygiene, environmental cleaning, visitor access and monitoring and managing personnel. The increase of awareness of personal protection, sufficient PPE and proper preparedness and response as recommended in the guidelines play an important role in reducing the risk of infection for doctors and other HCWs.

In COVID-19 pandemic, older people and people with chronic co-morbidities are reported to have the highest risk of dying from the virus. But doctors are also particular at risk because they are overworked, exposed to many infected pa- tients and may lack adequate protection [28]. The doctors' deaths in the current COVID-19 pandemic highlighted an unfortunate reality of any new and evolving outbreak: Doctors and healthcare workers on the frontlines face a higher risk of contracting the infection.

\section{Financial Support}

None.

\section{Ethical Approval}

Not required.

\section{Disclosure Statement}

The author declares that he has no conflicts of interest related to the submitted paper.

\section{References}

1. WHO (2020) Pneumonia of unknown cause - China.

2. WHO (2020) Statement regarding cluster of pneumonia cases in Wuhan, China.

3. Zhu N, Zhang D, Wang W, et al. (2020) A novel coronavirus from patients with pneumonia in China, 2019. N Engl J Med 382: 727-733.

4. Gaunt ER, Hardie A, Claas EC, et al. (2010) Epidemiology and clinical presentations of the four human coronaviruses 229E, HKU1, NL63, and OC43 detected over 3 years using a novel multiplex real-time PCR method. J Clin Microbiol 48: 2940-2947.

5. Li Q, Guan X, Wu P, et al. (2020) Early transmission dynamics in Wuhan, China, of novel coronavirus-infected pneumonia. N Engl J Med 382: 1199-1207.

6. Rothe C, Schunk M, Sothmann P, et al. (2020) Transmission of 2019-nCoV infection from an asymptomatic contact in Germany. N Engl J Med 382: 970-971.

7. WHO (2020) Statement on the second meeting of the International Health Regulations(2005) Emergency Committee regarding the outbreak of novel coronavirus (2019-nCoV).

8. WHO (2020) Naming the coronavirus disease (COVID-19) and the virus that causes it.

9. WHO (2020) Director-general's opening remarks at the media briefing on COVID-19.

10. Novel Coronavirus Pneumonia Emergency Response Epidemiology Team (2020) Vital surveillances: The epidemiological characteristics of an outbreak of 2019 novel coronavirus diseases (COVID-19)-China, 2020.

11. Wu Z, McGoogan JM (2020) Characteristics of and important lessons from the coronavirus disease 2019 (COVID-19) outbreak in China: Summary of a report of 72314 cases from the Chinese Center for Disease Control and Prevention. JAMA.

12. Liu K, Fang YY, Deng Y, et al. (2020) Clinical characteristics of novel coronavirus cases in tertiary hospitals in Hubei Province. Chin Med J (Engl).

13. Koh D (2020) Occupational risks for COVID-19 infection. Occup Med (Lond) 70: 3-5.

14. Wang D, Hu B, Hu C, et al. (2020) Clinical characteristics of 138 hospitalized patients with 2019 novel coronavirus-infected pneumonia in Wuhan, China. JAMA.

15. (2020) In China, health care workers are dying of coronavirus-and exhaustion. Advisory Board. 
16. (2020) Coronavirus live updates (Sunday): China discovers infected travelers from Iran and UK, another Wuhan doctor dies. Caixing Global.

17. (2020) More than 60 doctors in Italy have died in COVID-19 Pandemic. Medscape.

18. (2020) Six Indonesian doctors die from COVID-19, cases exceed 500. Jakarta Globe.

19. (2020) Nurses' Union Survey found hospitals are unprepared for coronavirus.

20. (2020) Americans are hoarding face masks to prepare for coronavirus. Big Think.

21. WHO (2015) Health worker Ebola infections in Guinea, Liberia and Sierra Leone. Preliminary report.

22. Kilmarx PH, Clarke KR, Dietz PM, et al. (2014) Ebola virus disease in health care workers--Sierra Leone. MMWR Morb Mortal Wkly Rep 63: 1168-1171.
23. Wong TW (2003) An outbreak of SARS among healthcare workers. Occup Environ Med 60: 528.

24. (2020) Health workers fighting coronavirus in China die of infection, fatigue. Los Angeles Times.

25. (2020) Coronavirus: Doctors collapse from exhaustion as virus spreads through South Korea. The Telegraph.

26. (2020) Managing healthcare workers' stress associated with the COVID-19 virus outbreak-PTSD. National Center for PTSD.

27. Verma S, Mythily S, Chan YH, et al. (2004) Post-SARS psychological morbidity and stigma among general practitioners and traditional Chinese medicine practitioners in Singapore. Ann Acad Med Singapore 33: 743-748.

28. Wang J, Zhou M, Liu F (2020) Exploring the reasons for healthcare workers infected with novel coronavirus disease 2019 (COVID-19) in China. J Hosp Infect. 